\title{
Improved the expression level of active transglutaminase by directional increasing copy of $m t g$ gene in Pichia pastoris
}

\author{
Xiaoping Song ${ }^{1,3^{*}} \mathbb{D}$, Changsheng Shao ${ }^{3}$, Yugang Guo ${ }^{2,3}$, Yajie Wang ${ }^{1}$ and Jingjing Cai ${ }^{1}$
}

\begin{abstract}
Background: The microbial transglutaminase (MTG) is inactive when only the mature sequence is expressed in Pichia pastoris. Although co-expression of MTG and its N-terminal pro-peptide can obtain the active MTG, the enzyme activity was still low. One of the basic steps for strain improvement is to ensure a sufficient level of transcription of the heterologous gene, based on promoter strength and gene copy number. To date, high-copynumber recombinants of $P$. pastoris are achievable only by cloning of gene concatemers, so methods for rapid and reliable multicopy strains are therefore desirable.

Results: The coexpression strains harboring different copies $m t g$ were obtained successfully by stepwise increasing Zeocin concentration based on the rDNA sequence of $P$. pastoris. The genome of coexpression strains with the highest enzyme activity was analyzed by real-time fluorescence quantitative PCR, and three copies of $m$ tg gene ( $m$ tg-3c) was calculated according to the standard curve of gap and $m$ tg genes (gap is regarded as the single-copy reference gene). The maximum enzyme activity of $m t g-3 c$ was up to $1.41 \mathrm{U} / \mathrm{mL}$ after being inducted for $72 \mathrm{~h}$ in $1 \mathrm{~L}$ flask under optimal culture conditions, and two protein bands were observed at the expected molecular weights ( $40 \mathrm{kDa}$ and $5 \mathrm{kDa}$ ) by Western blot. Furthermore, among the strains detected, compared with $m t g-2 \mathrm{c}, \mathrm{mtg}-6 \mathrm{c}$ or $m t g-8 c, m t g-3 c$ is the highest expression level and enzyme activity, implying that $m t g-3 c$ is the most suitable for co-expression pro-peptide and MTG.
\end{abstract}

Conclusions: This study provides an effective strategy for improving the expression level of active MTG by directional increasing of $m t g$ copies in P. pastoris.

Keywords: Transglutaminase, Pichia pastoris, rDNA, Pro-peptide, Co-expression

\section{Background}

Transglutaminase (TG, EC2.3.2.13) is an effective protein cross-linker that transfers acyl between glutamine and acyl acceptors to improve the properties of proteins [1-3]. The microbial transglutaminase (MTG) is widely used in the food industry to improve the texture and nutrition $[4,5]$. Besides, TG has broad potential applications in biopharmaceuticals [6-9],tissue engineering [3, 7], sitedirected protein cross-linking $[1,3,10]$, and antibodydrug conjugates [11].

\footnotetext{
* Correspondence: sxp20081012@sina.com

'Department of Pharmacy, Anhui Medical College, Hefei 230061, China

${ }^{3}$ Anhui Engineering Research Center of Recombinant Protein Pharmaceutical Biotechnology, Hefei 230022, China

Full list of author information is available at the end of the article
}

The MTG was produced by Streptomyces mobaraense [12], Streptomyces hygroscopicus [13], and Bacillus circulans [14]. The catalytic active center of MTG consisted of catalytic triad C64-D255-H274 is located at the bottom of its active fracture (Additional file 1: Figure S1). The MTG could be secret to extracellular as a zymogen and the $\mathrm{N}$-terminal peptide was removed by endogenous protease to activate the enzyme $[15,16]$. It is hard to express MTG in engineering strains due to the activation mode [17-21]. Some researchers suggested that the MTG is inactive if the full or mature sequence is expressed in P. pastoris $[17,21]$. To solve the problem, the pro-peptide and mature sequence of MTG could be expressed as two separate elements in $P$. pastoris. The activity of MTG reached $0.25 \mathrm{U} / \mathrm{mL}$ and $0.338 \mathrm{U} / \mathrm{mL}$ respectively in flasks [22], indicating that the pro-peptide

(c) The Author(s). 2019 Open Access This article is distributed under the terms of the Creative Commons Attribution 4.0 International License (http://creativecommons.org/licenses/by/4.0/), which permits unrestricted use, distribution, and 
sequence could promote and activate the mature enzyme. However, the activity was too low to meet the industrial requirements. Therefore, the increasing demand on MTG is urgently needed to improve the enzyme activity of MTG.

As we know, the yield of protein is related to the copy number of the target gene [23, 24]. This is a useful method to improve the yield of protein by increasing the copy number of the target gene in yeast $[25,26]$. The repeated sequence of rDNA of $P$. pastoris was separated by the non-transcribed intergenic spacer (NTS) [26, 27]. It could be used as the recombination site to increase the copy number of the target gene. In our previous work, the gene of snake venom antiplatelet thrombolytic was integrated into the rDNA repeat sequence. The combinant antiplatelet thrombolytic shared similar physicochemical properties and biological activities with the natural antiplatelet thrombolytic [27]. Therefore, the strategy based on the repeated sequence of rDNA of $P$. pastoris to construct multiple copy of the target gene can be employed in this paper.

In order to improve the expression level and the enzyme activity of the recombinant MTG, genes coding MTG and pro-peptide ( $m$ tg and pro) were optimized to make their mRNA secondary structure more stable according to GC contents and the codon bias of $P$. pastoris. The genes (pro and $m t g$ ) were inserted sequentially into the chromosome of $P$. pastoris at ribosomal DNA repeat sites (rDNA) and the mutant histidinol dehydrogenase site (His 4), respectively, and regulated by the constitutive promoter (GAP) and the methanol-induced promoter $(A O X 1)$, respectively. A high coexpression of pro-peptide and MTG was obtained by high-pressure screening of antibiotic and optimization of fermentation conditions. To our knowledge, this is a new report of improving the expression level of active MTG by directional increase of the copy of $m t g$ gene in P. pastoris.

\section{Results \\ Construction balanced co-expression strains for pro- peptide and MTG}

The genes (pro and $m t g$ ) were inserted into vector pGAP9 and pPICZ $\alpha$ to construct the expression vector pGAP9-proand pPICZ $\alpha$-rDNA-mtg, respectively (Fig. 1). Both pro and $m$ tg genes could be integrated into the his4 site and non-coding rDNA sequence site when vectors were transformed into the host strain GS115, respectively. The genes (pro and $m t g$ ) were fused with the $S$. cerevisiae $\alpha$-mating factor signal sequence and placed under the regulation of GAP and AOX1 promoters. The recombinants of co-expression strain GS115 (pro/rDNA$m t g$ ) were analyzed by PCR (Additional file 4: Figure S4), of which the results implied that the pro and $m t g$ genes were integrated into the desired location of $P$. pastoris genome.

To generate the coexpression strain of pro-peptide and mature MTG, the expression plasmid (pGAP-pro) was transformed into P. pastoris GS115 and selected by histidine-deficient minimal dextrose (MD) plates, while the clone (\# 34-4) with the highest expression of propeptide was selected (Fig. $2 \mathrm{a}$ and $\mathrm{b}$ ) and used for the host strain of the co-expressed (pro/rDNA-mtg). The clones with higher co-expression were selected in $\mathrm{MD}$ plate (contain $400 \mu \mathrm{g} / \mathrm{mL}, \quad 600 \mu \mathrm{g} / \mathrm{mL}, \quad 800 \mu \mathrm{g} / \mathrm{mL}$

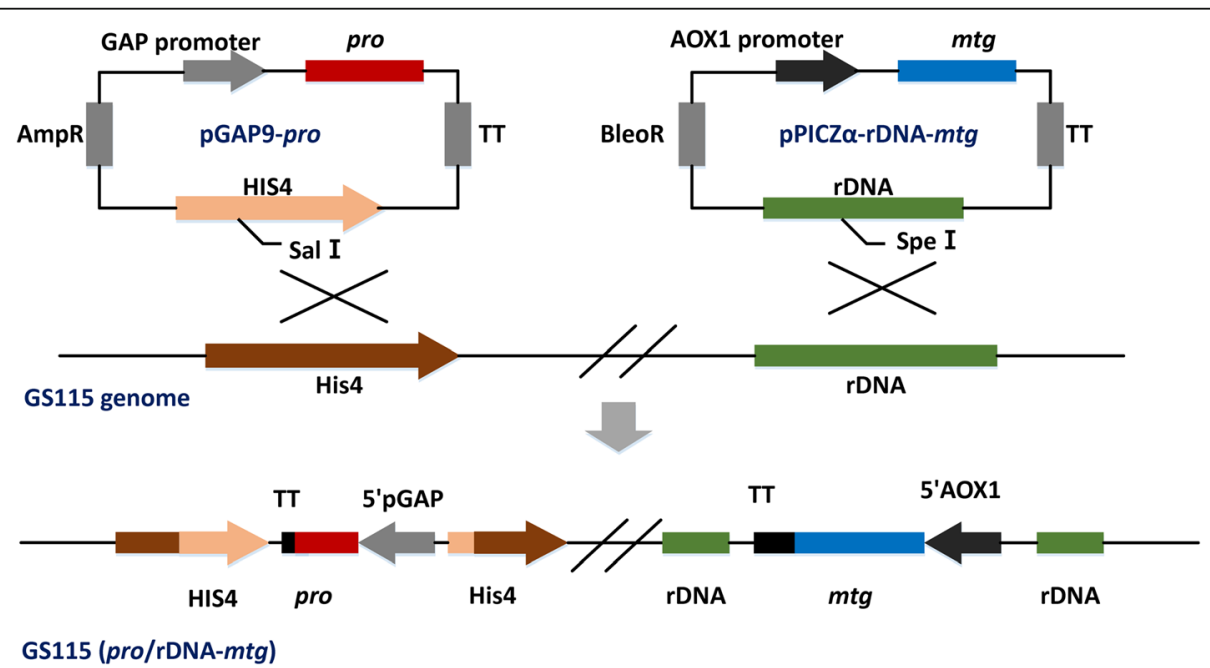

Fig. 1 Strategy for the construction of $m t g$ and pro co-expression strains. This schematic map represents the constructed expression vectors for the pro and $m t g$, designated pGAP9/pro and pPICZa-rDNA/mtg, respectively. The his4 and rDNA non-coding sequences allow the vector to be inserted into the corresponding sites in the genome of strain GS115 through homologous recombination. The pPICZa-rDNA-mtg vector was constructed by introducing a non-coding rDNA (indicated as rDNAnc) sequence into the pPICZa vector. The expression of both pro and $m$ tg were under the control of the GAP and AOX1 promoters, respectively 


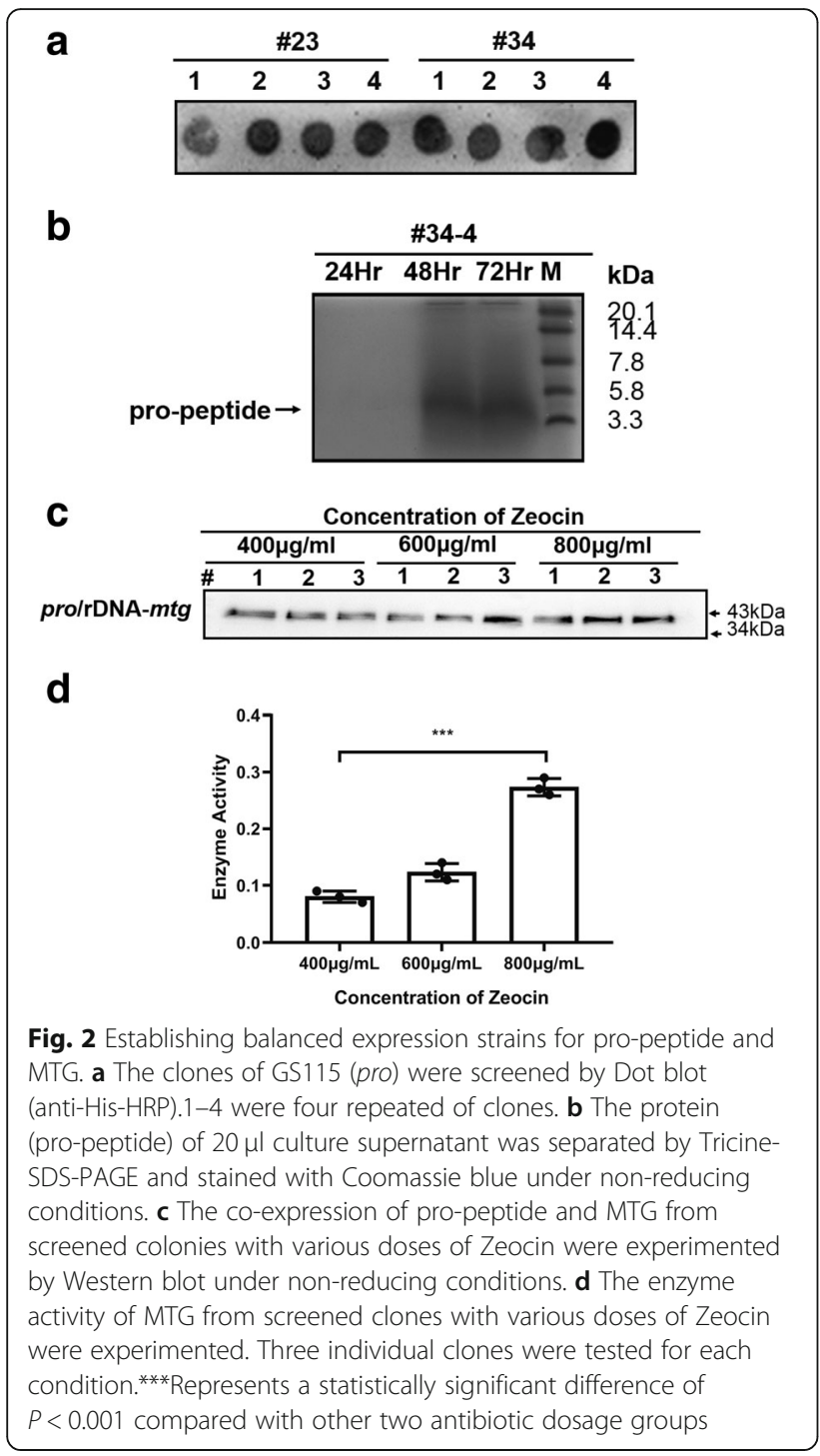

Zeocin) and the expression of MTG was measured by Western blot with anti-MTG antibody employed. All selected clones rendered positive results by Western blot and showed an increasing trend with the increase of antibiotic dose (Fig. 2c). The enzyme activity of clone (contain $800 \mu \mathrm{g} / \mathrm{mL}$ Zeocin) reached up to $0.27 \mathrm{U} / \mathrm{mL}$, higher than those of the other two antibiotic dosage groups $(0.08 \mathrm{U} / \mathrm{mL}, 0.12 \mathrm{U} / \mathrm{mL}$ ) (Fig. $2 \mathrm{~d}$ ).

\section{Copy number of $m t g$ gene in $P$. pastoris genome}

The results of $m t g$ copy number in $P$. pastoris genome are shown in Table 1. The amplification and melt curves of gap and $m$ tg genes are shown in Fig. 3 while the standard curves are shown in Additional file 5: Figure S5. Both the melt curves of gap and $m$ tg genes have only one single peak, indicating specificity of the PCR products (Fig. 3a, b). The amplification curves of gap and $m t g$ genes were shown in Fig. 3c and d. The regression equations of both gap and $m$ tg genes standard curve were $\mathrm{y}=-3.6892 \mathrm{x}$ $+35.747(\mathrm{R}=0.9985), \mathrm{y}=-3.4511 \mathrm{x}+33.968(\mathrm{R}=0.9985)$, respectively. By the slope of the two standard curves, the response efficiency of gap and $m t g$ genes were calculated as 86.66 and $94.88 \%$, respectively, indicating similar results of the two ones. The result of $m t g$ gene copy number in $P$. pastoris (with the highest activity) is shown in Table 1 , demonstrating that $m t g$ gene existed in the genome in the form of 3.36 copies and that 3 copies $m t g$ gene were directed to the rDNA site in $P$. pastoris and named $m t g-3 \mathrm{c}$. In addition, the copy numbers of $m t g$ gene in the other three detected strains were $2.21,5.72$ and 7.62, respectively (named $m t g-2 \mathrm{c}, m t g-6 \mathrm{c}, m t g-8 \mathrm{c}$ ) (Table 1).

\section{Effects of temperature and $\mathrm{pH}$ on growth and enzyme activities of co-expressing strains}

The engineering strain $m t g$-3c (pro/rDNA- $m t g$ ) was cultured in $200 \mathrm{~mL}$ BMMY medium at different $\mathrm{pH}$, with the result suggesting that the initial $\mathrm{pH}$ of the medium exerted minor influence on the cell growth but significant one on the enzyme activity of MTG as illustrated in Fig. 4a, b. The activity of MTG was higher at $\mathrm{pH} 6$ and 7 than that at $\mathrm{pH} 5$ or 8 , indicating that the neutral environment was conducive to producing enzymes or the enzyme was more stable in neutral environment. Furthermore, the strain GS115 (pro/rDNA-mtg) was inducted at various temperatures as shown in Fig. 4c, d. The activity of MTG at 25 or $30^{\circ} \mathrm{C}$ was higher than that at $20^{\circ} \mathrm{C}$ within $48 \mathrm{~h}$. However, the activity of MTG at $30^{\circ} \mathrm{C}$ decreased after $48 \mathrm{~h}$. The highest activity of MTG was $0.91,1.12$, and $0.81 \mathrm{U} / \mathrm{mL}$ at 20,25 , and $30^{\circ} \mathrm{C}$, respectively for $72 \mathrm{~h}$, indicating that the optimum inducted temperature was $25^{\circ} \mathrm{C}$. The activity of MTG would increase significantly by controlling the fermentation conditions (the initial $\mathrm{pH}$ of BMMY medium and inducted temperatures).

\section{Co-expression of pro-peptide and MTG in $1 \mathrm{~L}$ flask}

The engineering strain $m t g-3 \mathrm{c}$ was inoculated into BMGY medium for $24 \mathrm{~h}$ and then transferred into 200 $\mathrm{mL}$ BMMY medium. The pro-peptide and MTG in the supernatant have been evaluated by SDS-PAGE electrophoresis and Western blot, respectively (Fig. 5). The experimental results showed that one protein band was observed at the expected molecular weight (40 $\mathrm{kDa}$ ) (Fig. 5d). In addition, another protein band was observed at the expected molecular weight $(5 \mathrm{kDa})$ by Tricine-SDS-PAGE (Fig. 5b). Because the molecular weights of pro-peptide $(5 \mathrm{kDa})$ and MTG $(40 \mathrm{kDa})$ are significantly different from each other, it is difficult to display them on the same SDS-PAGE gel (Fig. 5a, c). The strategy of combining two homologous recombination sites (His4 and rDNA repeat site) could enable the co-expression of pro-peptide and MTG in 
Table 1 Copy numbers of gap and $m$ tg gene detected by real-time fluorescent quantitative PCR

\begin{tabular}{|c|c|c|c|c|c|}
\hline \multirow[t]{2}{*}{ Strain } & \multicolumn{2}{|l|}{ Value $\mathrm{Ct}$} & \multicolumn{2}{|c|}{ Gene copy $\left(10^{n}\right)$} & \multirow{2}{*}{$\begin{array}{l}\text { Number of copies of mtg gene } \\
\text { in P. Pichia genome (mtg copy } \\
\text { number/gap copy number) }\end{array}$} \\
\hline & mtg gene & gap gene & mtg gene & gap gene & \\
\hline 1 & $14.14 \pm 0.27$ & $16.49 \pm 0.17$ & $5.58 \pm 1.02$ & $1.66 \pm 0.19$ & $3.36 \pm 0.24$ \\
\hline 2 & $14.93 \pm 0.28$ & $16.67 \pm 0.22$ & $3.29 \pm 0.50$ & $1.48 \pm 0.18$ & $2.21 \pm 0.11$ \\
\hline 3 & $14.97 \pm 0.30$ & $18.23 \pm 0.23$ & $3.20 \pm 0.63$ & $5.60 \pm 0.71$ & $5.72 \pm 0.35$ \\
\hline 4 & $15.30 \pm 0.41$ & $19.05 \pm 0.17$ & $2.56 \pm 0.55$ & $3.36 \pm 0.31$ & $7.62 \pm 1.11$ \\
\hline
\end{tabular}

Datas are presented as mean \pm SD of triplicate observations. Strain 1: the clone with the highest enzyme activity. Strain 2,3,4:another three positive clones

P. pastoris. The enzyme activity of $m t g-3 c$ (culture supernatant) was $1.41 \mathrm{U} / \mathrm{mL}$ after being inducted for $72 \mathrm{~h}$.

\section{Discussion}

The $P$. pastoris has many outstanding advantages as a host for the expression of foreign proteins $[2,18]$. It enjoys the advantages of the strong inducing promoter AOX1, high protein expression, stable genetic traits, simple composition of the medium, etc. Various exogenous genes such as interferon, HBsAg, phytase, albumin, antibody and collagen were expressed in $P$. pastoris successfully $[18,21,27]$. Some researchers have attempted to express recombinant Streptomyces MTG in P. pastoris; however, the yield of MTG is very low $[2,18,21]$. We also encountered the problem of low yield of recombinant MTG in the previous research. In order to increase the yield of recombinant MTG in P. pastoris, we constructed 3 copies $m t g$ of GS115 (pro/rDNA- $m t g$ ) with higher expression, and optimized such conditions of protein expression as $\mathrm{pH}$ and temperature to increase the yield of MTG.

The copy number of the target gene is an important factor for the yield of the target protein in P. pastoris [23]. The expression of the target protein could be effectively boosted by increasing the copy number to $4-9$ of the gene [17]. When the copy number was more than nine, the yield of target gene might decrease because the
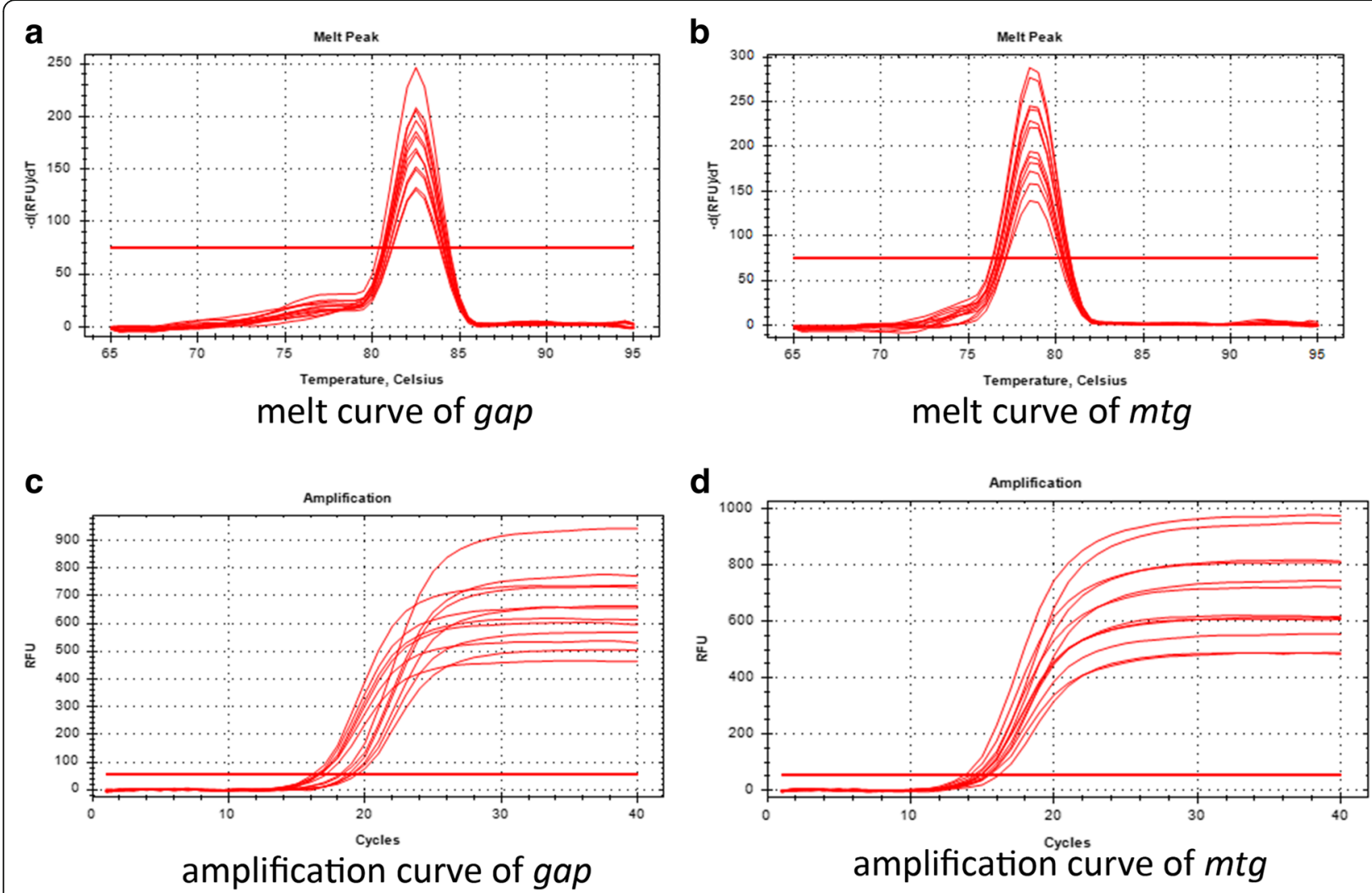

Fig. 3 Detection of $m$ tg copy number in P. pastoris genome by absolute quantitative PCR. a and $\mathbf{b}$ The melt curves of gap and $m t g$ genes. $\mathbf{c}$ and d The amplification curves of gap and mtg genes 

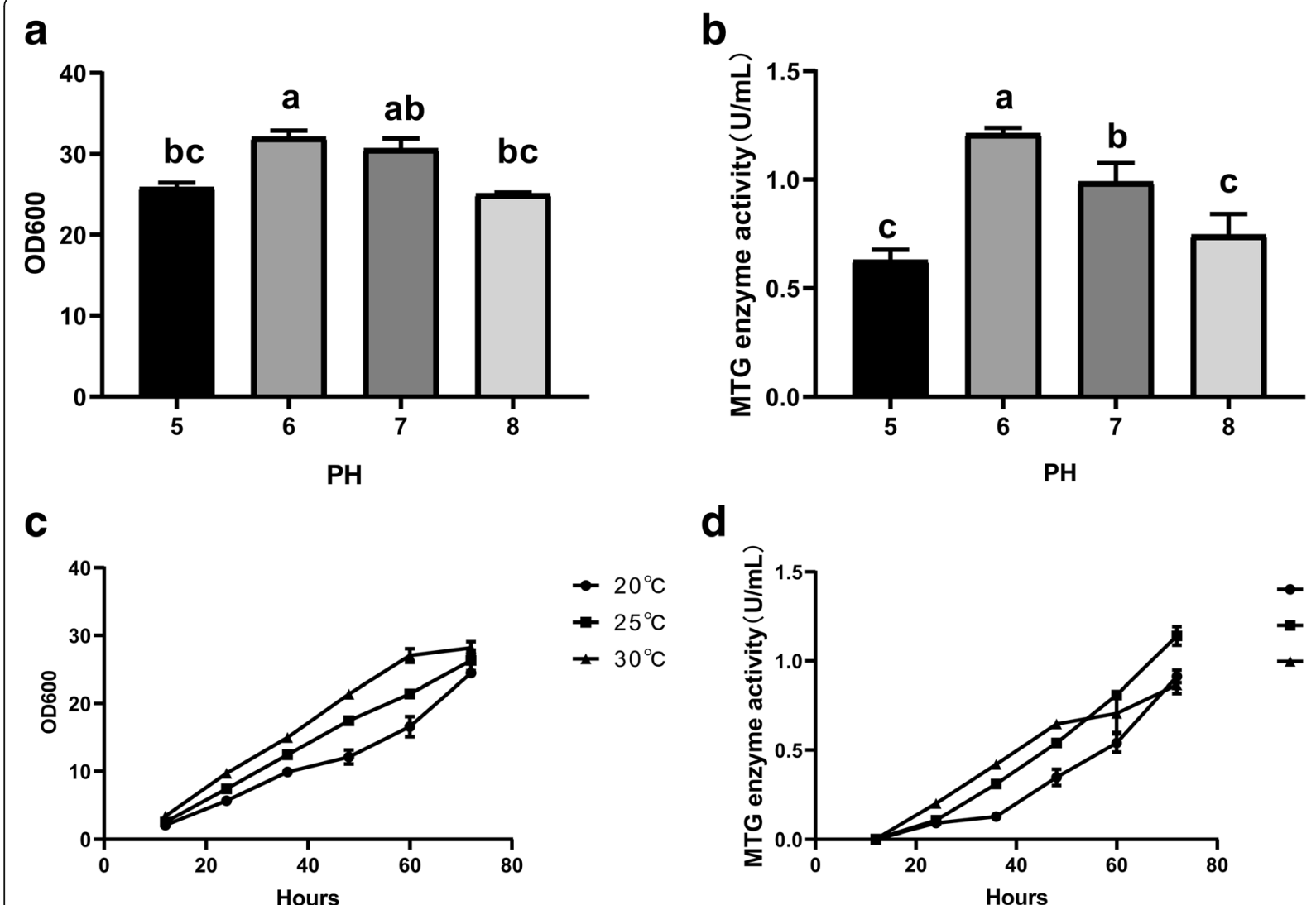

d

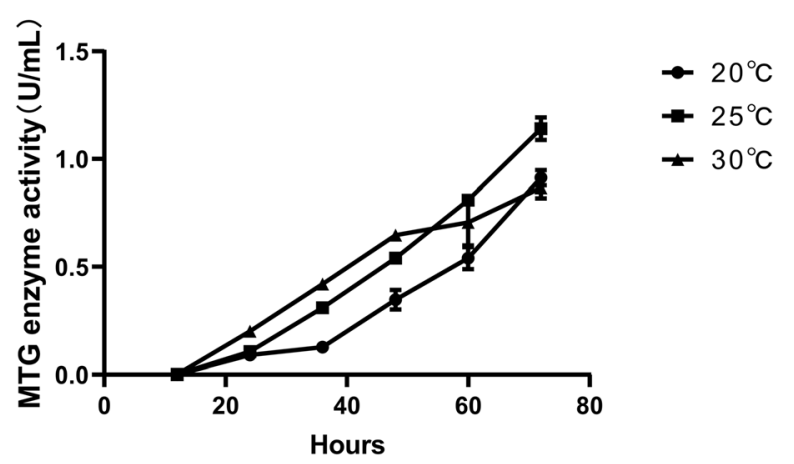

Fig. 4 Effects of inducted temperature and pH on growth of GS115 (pro/rDNA-mtg) and enzyme activities of MTG. a and $\mathbf{b}$ were the effect of different $\mathrm{pH}$ on the strain growth and enzyme activity of MTG. $\mathbf{c}$ and $\mathbf{d}$ were the effect of different induced temperature on the growth and enzyme activity of MTG. Datas of strain growth and enzyme activity are presented as mean \pm SD of triplicate observations. Alphabets (a-c) in superscript donate significant difference $(P<0.05)$

high concentration of target protein had a negative effect on the strain growth and metabolism $[25,28]$. In this study, we constructed a high expression strain harboring different copies $m t g$ by directionally increasing $m t g$ gene copy in P. pastoris, and verified the influence of gene dosage on the expression level of MTG. The absolute quantitative PCR analysis was successfully applied to quantify $m t g$ copies in the recombination strains. Among the clones being detected, $m t g$ genes existed in the $P$. pastoris genome in the form of 2,3,6 or 8 copies, respectively (named $m t g-2 \mathrm{c}, m t g-3 \mathrm{c}, m t g-6 \mathrm{c}, \quad m t g-8 \mathrm{c}$ ). Under the same culture conditions (200mLBMMY in shake flask), the effect of different copy $m t g$ genes on protein expression and enzyme activity was shown in Additional file 6: Table S1 and Figure S6. The experimental datas showed that the expression levels of $m t g$ gene varying with copy numbers were inconsistent, and fell into the range of was $m t g-3 c>m t g-2 c>m t g-6 c$ (Additional file 6: Figure S6). The results revealed that the recombinant strain produced the enzyme faster in case of no more than 3 copies of $m t g$ gene, showing the positive effect of gene copy number. However, when gene copy number is more than 3 , the enzyme production of the strain dwindled for the excessive protein load, resulting in more heteropolymeric protein and a sharp decrease in enzyme activity than one of the low-copy strain $[28,29]$. This fact demonstrated the negative effect of gene copy number, which may be attributed to the burden of the excessive copy number on the cell, resulting in the metabolic imbalance of the strain itself. For this reason, the multiple copies may not result in a high expression level. These results imply that other factors limited the transcription, translation, and secretion of MTG, indicating that the copy number was not necessarily related to the expression level, and that the reasonable copy number was beneficial to improving the protein expression level. These results revealed that 3 copies of $m t g$ gene were the most suitable for pro and $m$ tg co-expression in this study.

The result of culture conditions optimization showed that the initial $\mathrm{pH}$ of medium has significant influence on the enzyme activity of MTG. The optimum $\mathrm{pH}$ of MTG expression is between 6.0 and 7.0 and the stability of MTG is poor under acid and alkaline conditions, 


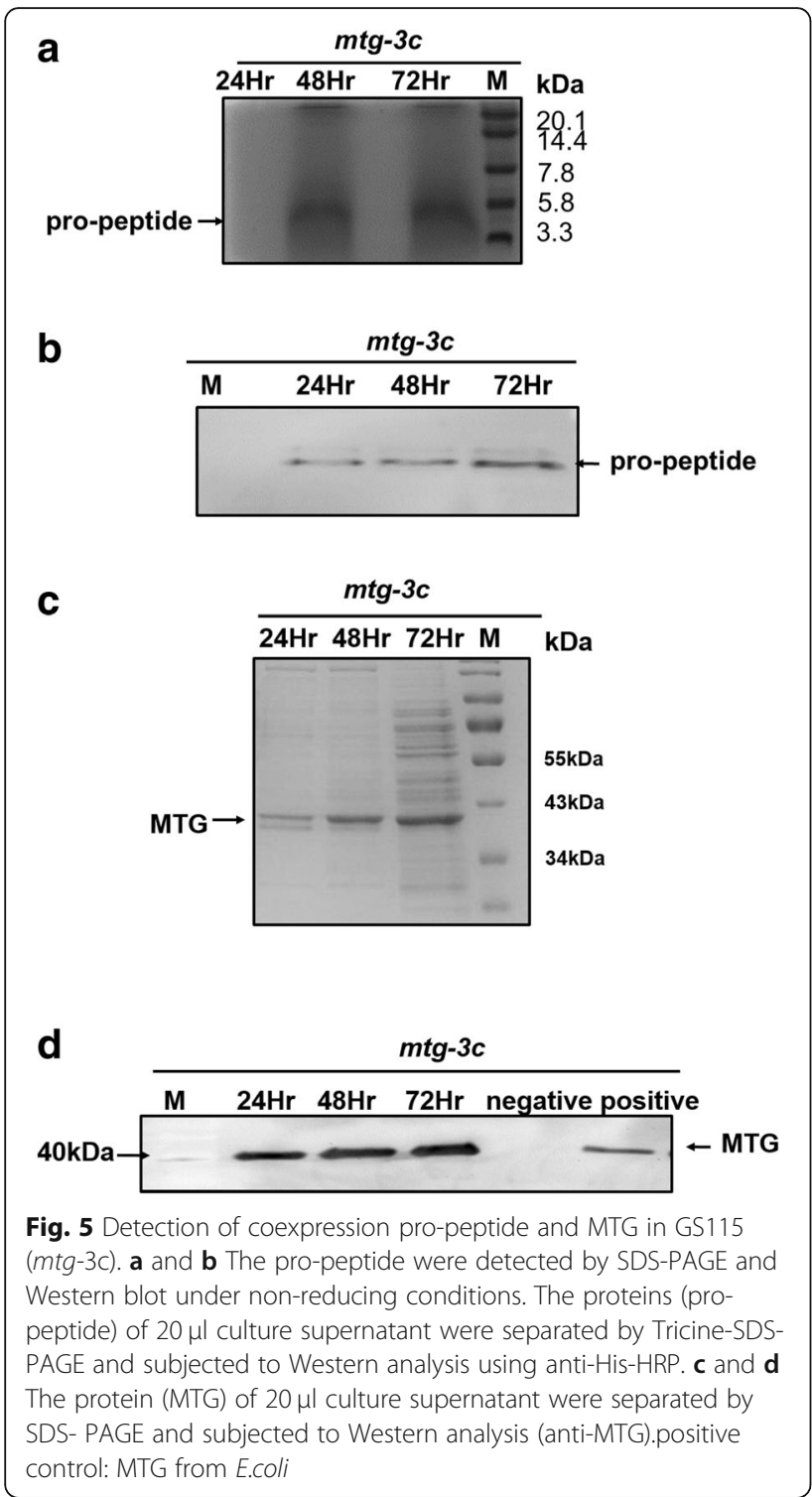

especially under acid conditions $(\mathrm{pH}<6.0)$. The activity of protease produced by $P$. pastoris is suppressed at the $\mathrm{pH} 6.0-7.0$, which could reduce the degradation of the target protein [28]. Furthermore, the temperature would affect the yield of MTG, which is lower at $30^{\circ} \mathrm{C}$ than that at 20 or $25^{\circ} \mathrm{C}$, indicating that the rate of cell growth is lower at 20 and $25^{\circ} \mathrm{C}$, but the yield of MTG is higher in this case, attributable to the hypothermia reducing the residence time of the protein in the endoplasmic reticulum, which is conducive to the correct folding of the target protein [30, 31].

Finally, the engineering strain GS115 (pro/rDNA-mtg) was cultured in $200 \mathrm{~mL}$ BMMY medium for $72 \mathrm{~h}$. The maximum enzyme activity of supernatant was $1.41 \mathrm{U} / \mathrm{mL}$, 3.17 times higher than that of a coexpression strain as single-copy expression cassettes $(0.338 \mathrm{U} / \mathrm{mL})$ [22]. In addition, two protein bands were observed at the expected molecular weight ( $40 \mathrm{kDa}$ and $5 \mathrm{kDa}$ ) by Western blot, indicating that the co-expression of pro-peptide and MTG can directly secrete active MTG. This is a new report of improving the expression level of active MTG by directional increasing copies of $m t g$ gene in $P$. pastoris in the published articles .

The pro-peptide is essential for protein folding, playing the main roles of helping proteins form compact structures and thus reducing the steric hindrance of proteins, guiding proteins to correctly fold and forming active mature enzymes [21]. In this study, the expression level of MTG was mainly measured by enzyme activity. Therefore, the enhanced expression of pro-peptide will facilitate the correct folding of MTG, thus forming much more active MTG. However, the balance of coexpression of pro-peptide and MTG is a problem to be solved in this study. The balance expression of MTG and pro-peptide can be achieved by gradually increasing the concentration of Zeocin antibiotics.

\section{Conclusions}

Based on the non-transcriptional rDNA sequence of P. pastoris, the co-expression strain (pro/rDNA-mtg) with three copies $m t g$ genes was constructed successfully. However, the expression in P. pastoris is at the milligram per liter level in this paper, which needs to be raised to grams per liter for industrial applications. Successful co-expression of MTG and pro-peptide as well as optimization of their ratios is a challenge, but worth investigating.

\section{Methods}

Strains and vectors

Strains and vectors were described in Table 2. The E. coli was cultured in Luria-Bertani (LB) medium at $37^{\circ} \mathrm{C}$, whereas the P. pastoris was cultured in buffered glycerolcomplex medium (BMGY) or buffered methanol-complex medium (BMMY) at $28^{\circ} \mathrm{C}$ or $25^{\circ} \mathrm{C}$, respectively.

\section{Synthesis of $m t g$ and pro genes}

The $m t g$ and pro genes were selected from the cDNA of S.mobaraensis (NO. DQ132977) [19, 21] and synthesized after the codon optimization based on the P. pastoris preference (Additional file 2 and Additional file 3: Figures S2 and S3). The primers were designed by Primer premier 5(http://www.premierbiosoft.com/primerdesign/index.html) as shown in Table 3.

The restriction enzyme site (XhoI) and the Kex2 endopeptidase recognition site were added to the $5^{\prime}$ terminus of MTG and pro-peptide (primer P1 and P3). The restriction enzyme site of NotIand the sequence of histidine tag were added to the 3 '-terminus (primer P2 and P4). 
Table 2 Strains and plasmids used in this study

\begin{tabular}{|c|c|c|}
\hline Strains & Genotype & References \\
\hline E. coli TOP10 & 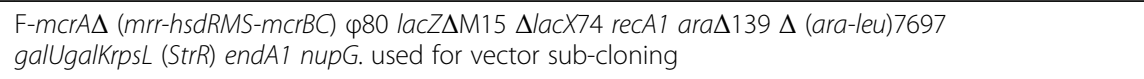 & Invitrogen \\
\hline Pichia pastoris GS115 & his4,host strain & Invitrogen \\
\hline GS115 (pro) & The pro-peptide of MTG was integrated into his4 site of Pichia pastoris GS115 & This study \\
\hline GS115(pro/rDNA-mtg) & The mature sequence of MTGwas integrated into non-coding rDNA site ofGS115 (pro) & This study \\
\hline Plasmids & Description & Reference \\
\hline pPICZa-B & Containing AOX1 promoter for tightly regulated, methanol-induced expression of the gene, Zeocin ${ }^{R}$ & Invitrogen \\
\hline pGAP9 & pPIC9 derivative, GAP promoter instead of AOX1 promoter & Lab stock \\
\hline pGAP9-pro & pGAP9 derivative carrying an internal $135 \mathrm{bp}$ fragment of pro gene & This study \\
\hline pPICZa-rDNA-mtg & pPICZa-mtg derivative carrying an internal 1153 bp fragment of rDNA gene, Zeocin ${ }^{R}$ & This study \\
\hline
\end{tabular}

The construction of rDNA-mediated $m t g$ multi-copy expression vector

The structure of expression pPICZ $\alpha$-rDNA-mtg was shown in Fig. 1.The AOX1-terminator (primer P5and P6) and non-coding rDNA fragment (primer P7and P8) were amplified and fused to form the AOX1-rDNA by overlapping PCR as follows: a program consisting of 30 cycles at $95{ }^{\circ} \mathrm{C}$ for $40 \mathrm{~s}, 54{ }^{\circ} \mathrm{C}$ for $50 \mathrm{~s}$ and $72{ }^{\circ} \mathrm{C}$ for $120 \mathrm{~s}$. Then the AOX1-rDNA and the vector pPICZa-mtg were digested by NotI and BamHI and connected by T4 DNA ligase. The recombinant vector (pPICZ $\alpha$-rDNA-mtg) was transformed into $E$. coli TOP10 and sequenced to ensure base sequence was not mutated. The same

Table 3 PCR primers used in the present study

\begin{tabular}{|c|c|}
\hline Primer name & Primer sequence $\left(5^{\prime} \rightarrow 3^{\prime}\right)$ \\
\hline P1 & ACACTCGAGaaaagaGACTCCGACGACAGGGTCAC \\
\hline P2 & $\begin{array}{l}\text { TATGCGGCCGCTCAATGGTGATGGTGATGATGCGGC } \\
\text { CAGCCCTGC }\end{array}$ \\
\hline P3 & ACACTCGAGaaaagaGACAAT GGC GCG GGG GAAG \\
\hline P4 & $\begin{array}{l}\text { TATGCGGCCGCTCAATGGTGATGGTGATGATGGGGA } \\
\text { GCCCGGAACG }\end{array}$ \\
\hline P5 & TATGCGGCCGCCAGCTITCTAGAACAAAAACTCATCTC \\
\hline P6 & $\frac{\text { CTTAAATATTAGGAAAAACGGTAACCTTAT }}{\text { CTCACTTAATCTTCTGTACTCTG }}$ \\
\hline P7 & $\begin{array}{l}\text { CAGAGTACAGAAGATTAAGAGAGATAGT } \\
\text { TAGGTTACCGTITICCTAATATTAAG }\end{array}$ \\
\hline P8 & CGCGGATCCCTTCCACCAACAGTCAACCACCAGTC \\
\hline P9 & GGTATTAACGGTTTCGGACGTATTG \\
\hline P10 & GATGTTGACAGGGTCTCTCTCTTGG \\
\hline P11 & TGAAGAAAGAATTGGCTAACGG \\
\hline P12 & AGCTGGTCTGAAAGCATCTGG \\
\hline
\end{tabular}

Remarks: The underlined sites are those for the digestion of restriction enzymes Xhol. The wavy line sites are those for the digestion of restriction enzymes Notl. The double corrugated underlined sites are the ones for the digestion of restriction enzymes BamHI. 6xHis-tag label sequence is indicated by the dotted line. The Kex2-endopeptidase recognition site is marked with lowercase letters. The bold underlining represents the sequence of overlapping segments of the AOX1 terminator-rDNA fusion gene method was used to construct the control group (pPICZ $\alpha-m t g)$.

\section{Construction of the pro-peptide recombinant strain}

The plasmid pGAP9 and pro-peptide gene were digested with XhoI and NotI, ligated by DNA T4 ligase. Then it was transferred into E. coli TOP10 competent cells to construct the vector pGAP9-pro. It was digested by SalI and electro-transformed into host strain GS115. The recombinants were selected in MD plate without histidine and cultured in BMGY medium for $72 \mathrm{~h}$. The pro-peptide in the supernatant was identified by Dot blot with the antibody anti-His-HRP. Furthermore, the pro-peptide was measured by Tricine-SDS-PAGE. The engineering strain was named GS115(pro) and used as the host strain of co-expressed pro-peptide and MTG.

\section{Construction and selection of the co-expression strain}

Recombinant vectors ( $\mathrm{pPICZ} \alpha-\mathrm{rDNA}-m \mathrm{tg}$ ) were linearized by SpeI, and transformed into the host strain GS115 (pro-peptide). The clones with higher expression were selected by stepwise increasing the Zeocin concentration by $400 \mu \mathrm{g} / \mathrm{mL}, 600 \mu \mathrm{g} / \mathrm{mL}$ and $800 \mu \mathrm{g} / \mathrm{mL}$ on MD plates, respectively, so as to strike a better balance of the coexpression of MTG and pro-peptide. The clone with the highest expression was selected in MD plate (contain $800 \mu \mathrm{g} / \mathrm{mL}$ Zeocin) and named GS115 (pro/rDNA-mtg), respectively. Then they were cultured in BMGY medium for $24 \mathrm{~h}$ until the OD600 reached 6.0. The cell was harvested and induced in $5 \mathrm{~mL}$ BMMY medium containing $1 \%$ methanol for $72 \mathrm{~h}$. The MTG in the supernatant was identified by Western blot (the antibody: Anti-MTG) as described by "Molecular cloning: A laboratory manual". A clone with the highest enzyme activity was selected.

Analysis of $m$ tg copy number by absolute quantitative PCR The clones with the highest enzyme activity and other three positive ones were selected for analyzing copy number of $m t g$ gene, which in $P$. pastoris genome were 
determined by real-time fluorescent quantitative PCR (qPCR) [32]. The housekeeping gene gap exists as a single copy in the genome of P. pastoris [29], so the copy number of gap gene can be used to represent the initial copy number of the genome in the template.

The standard curves of gap and mtg genes were prepared with the standard plasmids containing gap and $m t g$ genes, respectively. Both gap and mtg genes were amplified by qPCR with the genome of P.pastoris transfected $m t g$ gene used as template, respectively (gap primer: P9 and P10, mtg primer: P11 and P12). Each sample was detected three times, and three parallel reactions were set for each time. The $C t$ values of gap and $m t g$ genes obtained substituted for the corresponding standard curves, and then the initial template copy number of gap and $m t g$ genes in DNA samples was calculated. The ratio of initial template copy number of $m t g$ and gap genes was the copy number of $m t g$ gene in $P$. pastoris genome. The reaction system is as follows: a total of $10 \mu \mathrm{l}$ reaction system, including SYRB Green Realtime PCR Master $5 \mu \mathrm{l}$, DNA template $1 \mu \mathrm{l}$, each primer $0.5 \mu \mathrm{l}\left(10 \mu \mathrm{mol} \cdot \mathrm{L}^{-1}\right), \mathrm{H}_{2} \mathrm{O} 3 \mu \mathrm{l}$. The reaction procedures: 10 mins at $95^{\circ} \mathrm{C}$, followed by 40 cycles of $10 \mathrm{~s}$ at $95^{\circ} \mathrm{C}, 30 \mathrm{~s}$ at $60^{\circ} \mathrm{C}$.

\section{Optimization of culture conditions for engineering strain pro/rDNA-mtg}

The strain GS115 (pro/rDNA-mtg) of higher enzyme activity was cultured in $100 \mathrm{~mL}$ BMGY medium for $24 \mathrm{~h}$ and controlled at $28^{\circ} \mathrm{C}$. Then the cell was harvested by centrifugation and inoculated into $200 \mathrm{~mL}$ BMMY medium at different initial $\mathrm{pH}(5.0,6.0,7.0$ and 8.0) for $72 \mathrm{~h}$ and maintained at $25^{\circ} \mathrm{C} .1 \mathrm{M} \mathrm{Na}_{2} \mathrm{HPO}_{4} \cdot 12 \mathrm{H}_{2} \mathrm{O}$ $(\mathrm{pH}=8.85)$ and $1 \mathrm{M} \mathrm{KH}_{2} \mathrm{PO}_{4}(\mathrm{pH}=4.01)$ were mixed in a certain proportion to form phosphate buffer with buffer range $\mathrm{pH} 4-9$ and used to adjust the initial $\mathrm{pH}$ of BMMY medium. The cell density (OD600) and the activity of MTG were detected as described below. Besides, the cell density and the activity of MTG were measured once every $12 \mathrm{~h}$ at different inducted temperatures (i.e. 20,25 and $30^{\circ} \mathrm{C}$ ).

The activity of MTG was measured as Yurimoto described [21]. The MTG enzyme activity was defined as follows: catalytic substrate (N-CBZ-Gln-Gly) to generate $1 \mu \mathrm{mol}$ glutamine hydroxamate (hydroxamic acid) at $37^{\circ} \mathrm{C}$ as one enzyme activity unit. Protein concentrations were determined by using a Bradford Protein Assay Kit (TaKaRa) and the standard protein was bovine serum protein (Thermo) [21].

\section{Expression of engineering strain pro/rDNA- $m$ tg in flask} The strain GS115 (pro/rDNA- $m t g$ ) of higher enzyme activity was cultured in $100 \mathrm{~mL}$ BMGY medium, as described above until the OD600 reached 6-7, and controlled at $28{ }^{\circ} \mathrm{C}$. Then they were harvested and cultured in $200 \mathrm{~mL}$ of BMMY medium containing 1\% methanol (initial OD600 reached 1.2-1.5) and maintained at $25^{\circ} \mathrm{C}$. $1 \%$ methanol $(V / V)$ was added into BMMY medium once every $24 \mathrm{~h}$ for continuous induction for $72 \mathrm{~h}$. The supernatant was collected once every $24 \mathrm{~h}$, centrifuged at $12000 \mathrm{~g}$ for $30 \mathrm{mins}$, and analyzed by SDS-PAGE and Western blot under non-reducing condition. The enzyme activity was detected and verified as described above.

\section{Additional files}

Additional file 1: Figure S1.Three-dimensional structure of MTG (a) and pro-MTG (b) from S. mobaraesis as determined by PyMOL. The catalytic triad C64-D255-H274 was represented by light blue, red and dark blue, respectively. (TIF $275 \mathrm{~kb}$ )

Additional file 2: Sequences of $m t g$ and pro genes. (DOCX $17 \mathrm{~kb}$ )

Additional file 3: Figures S2, S3. Secondary structure diagram of $m R N A$ of $m t g$ and pro. Figure S2. The secondary structure diagram of mRNA of $m t g-W T$ and $m t g-O p t$. Figure S3. The secondary structure diagram of mRNA of pro-WT and pro-Opt. (ZIP $93 \mathrm{~kb}$ )

Additional file 4: Figure S4. The product of gene amplification of pro and $m$ tg in GS115 (pro/ rDNA-mtg).1,3,5,7: pro was amplified by FWpro(P3) and Rv -pro (P4)primers; 2,4,6,8: mtg was amplified by Fw-mtg(P1) and 3'AOX primers; 9,10: negative controls; M: DNA Marker. (TIF 365 kb)

Additional file 5: Figure S5. Standard curves of gap and mtg gene. (DOCX 101 kb)

Additional file 6: Table S1, Figure S3. Effect on expression and enzyme activity of $m$ tg gene copies. Table S1. The MTG activity in different copy strains. Figure S6. Detection of protein expression in strains with different $m$ tg copy by Western blotting. The protein (MTG) in $20 \mu \mathrm{l}$ of culture supernatant were separated by Western blot analysis (anti-MTG). Lane 1-3: mtg-2c, lane 4-6: mtg-3c, lane 7-9: mtg-6c. (ZIP 48 kb)

\section{Abbreviations}

Ct: Cycle threshold; rDNA: ribosomal DNA; rDNAnc: non-coding rDNA; RFU: relative fluorescence units

\section{Acknowledgments}

We are thankful for the technical support for WB analysis from Anhui Engineering Research Center of Recombinant Protein Pharmaceutical Biotechnology; the kind gifts of pGAP9 and GS115 from Prof. Weihua Xiao, School of Life Sciences, University of Science and Technology of China, Hefei Anhui, China.

\section{Authors' contributions}

$X S, C S$ and $Y G$ conceived and designed the experiments. CS,YG, YW, and JC performed the experiments. XS,CS and YG analyzed the data. XS wrote the manuscript. All authors reviewed the manuscript.

\section{Funding}

This work was supported by the Anhui Provincial Natural Science Foundation (Grant No.1808085MC86), and the Education Science Foundation of Anhui Province (Grant Nos. KJ2016A378, KJ2017A695, KJ2015B02). These funding bodies had no role in the design of the study and collection, analysis, and interpretation of data and in writing the manuscript.

\section{Availability of data and materials}

The datasets supporting the conclusions of this article are included with in the article and its additional files. All strain materials were obtained from Anhui Medical College, Hefei, China.

Ethics approval and consent to participate Not applicable. 


\section{Consent for publication}

Not applicable.

\section{Competing interests}

The authors declare that they have no competing interests.

\section{Author details}

'Department of Pharmacy, Anhui Medical College, Hefei 230061, China. ${ }^{2}$ Institute of advanced technology, University of Science and Technology of China, Hefei 230031, China. ${ }^{3}$ Anhui Engineering Research Center of Recombinant Protein Pharmaceutical Biotechnology, Hefei 230022, China.

Received: 6 October 2018 Accepted: 5 July 2019

Published online: 30 July 2019

\section{References}

1. Shleikin AG, Danilov NP. Evolutionary-biological peculiarities of transglutaminase: Structure, physiological functions, application. J Evol Biochem Phys. 2011:47(1):1-14.

2. Li H, Zhang L, Cui Y, Luo X, Xue C, Wang S. Expression of soluble recombinant transglutaminase from Zea mays in Pichia pastoris. World J Microbiol Biotechnol. 2013;29(5):939-47.

3. Martins IM, Matos M, Costa R, Silva F, Pascoal A, Estevinho LM, Choupina AB. Transglutaminases: recent achievements and new sources. Appl Microbiol Biot. 2014:98(16):6957-64.

4. Gharibzahedi SMT, Chronakis IS. Crosslinking of milk proteins by microbial transglutaminase: utilization in functional yogurt products. Food Chem. 2018:245:620-32.

5. Kieliszek M, Misiewicz A. Microbial transglutaminase and its application in the food industry: a review. Folia Microbiol. 2014;59(3):241-50.

6. Verma A, Mehta K. Tissue transglutaminase-mediated chemoresistance in cancer cells. Drug Resist Update. 2007;10(4):144-51.

7. Garcia Y, Wilkins B, Collighan RJ, Griffin M, Pandit A. Towards development of a dermal rudiment for enhanced wound healing response. Biomaterials. 2008:29(7):857-68

8. Rosenthal AK, Derfus BA, Henry LA. Transglutaminase activity in aging articular chondrocytes and articular cartilage vesicles. Arthritis Rheum. 2014; 40(5):966-70.

9. Souza CFVD, Venzke JG, Flôres SH, Ayub MAZ. Enzymatic properties of transglutaminase produced by a new strain of Bacillus circulans BL32 and its action over food proteins. LWT Food Sci Technol. 2011:44(2):443-50

10. Mu D, Lu J, Shu C, Li H, Li X, Cai J, Luo S, Yang P, Jiang S, Zheng Z. Improvement of the activity and thermostability of microbial transglutaminase by multiple-site mutagenesis. Biosci Biotechnol Biochem. 2018:82(1):106-9.

11. Dennler P, Chiotellis A, Fischer E, Brégeon D, Belmant C, Gauthier L, Lhospice F, Romagne F, Schibli R. Transglutaminase-based chemoenzymatic conjugation approach yields homogeneous antibody-drug conjugates. Bioconjug Chem. 2014;25(3):569.

12. Sommer C, Hertel TC, Schmelzer CEH, Pietzsch M. Investigations on the activation of recombinant microbial pro-transglutaminase: in contrast to proteinase $\mathrm{K}$, dispase removes the histidine-tag. Amino Acids. 2012;42(2-3): 997-1006.

13. Cui L, Du GC, Zhang DX, Chen J. Thermal stability and conformational changes of transglutaminase from a newly isolated Streptomyces hygroscopicus. Bioresour Technol. 2008:99(9):3794-800.

14. Suzuki S, Izawa Y, Kobayashi K, Eto Y, Yamanaka S, Kubota K, Yokozeki K. Purification and characterization of novel transglutaminase from Bacillus subtilis spores. Biosci Biotechnol Biochem. 2000;64(11):2344-51.

15. Cai D, Wei X, Qiu Y, Chen Y, Chen J, Wen Z, Chen S. High-level expression of nattokinase inBacillus licheniformis by manipulating signal peptide and signal peptidase. J Appl Microbiol. 2016;121(3):704-12.

16. Cai D, Wang $H$, He P, Zhu C, Wang Q, Wei X, Nomura CT, Chen S. A novel strategy to improve protein secretion via overexpression of the SppA signal peptide peptidase in Bacillus licheniformis. Microb Cell Factories. 2017;16(1): $1-10$.

17. Javitt G, Ben-Barak-Zelas Z, Jerabek-Willemsen M, Fishman A. Constitutive expression of active microbial transglutaminase in Escherichia coli and comparative characterization to a known variant. BMC Biotechnol. 2017; 17(23):1-10
18. Liu S, Zhang D, Du G, Chen J. Progress in expression and molecular modification of microbial transglutaminase. Sheng Wu Gong Cheng Xue Bao. 2011;27(12):1681-9.

19. Salis B, Spinetti G, Scaramuzza S, Bossi M, Jotti GS, Tonon G, Crobu D, Schrepfer R. High-level expression of a recombinant active microbial transglutaminase in Escherichia coli. BMC Biotechnol. 2015;15(84):1-11.

20. Zhang L, Zhang L, Yi H, Du M, Ma C, Han X, Feng Z, Jiao Y, Zhang Y. Enzymatic characterization of transglutaminase from Streptomyces mobaraensis DSM 40587 in high salt and effect of enzymatic cross-linking of yak milk proteins on functional properties of stirred yogurt. J Dairy Sci. 2012; 95(7):3559-68.

21. Yurimoto H, Yamane M, Kikuchi Y, Matsui H, Kato N, Sakai Y. The propeptide of Streptomyces mobaraensis transglutaminase functions in cis and in trans to mediate efficient secretion of active enzyme from methylotropic yeasts. Biosci Biotechnol Biochem. 2004:68(10):2058-69.

22. Li P, Sun H, You L, Gong F, Chen Z, Zhang A, Zhu T. Direct secretory expression of active microbial transglutaminase in Pichia pastoris. Chin J Biotechnol. 2013;29(2):180-8.

23. Zhu T, Guo M, Tang Z, Zhang M, Zhuang Y, Chu J, Zhang S. Efficient generation of multi-copy strains for optimizing secretory expression of porcine insulin precursor in yeast Pichia pastoris. J Appl Microbiol. 2010; 107(3):954-63.

24. Khan FF, Carpenter D, Mitchell L, Mansouri O, Black HA, Tyson J, Armour JAL. Accurate measurement of gene copy number for human alphadefensin DEFA1A3. BMC Genomics. 2013;14.

25. Marx H, Mecklenbrauker A, Gasser B, Sauer M, Mattanovich D. Directed gene copy number amplification in Pichia pastoris by vector integration into the ribosomal DNA locus. FEMS Yeast Res. 2009;9(8):1260-70.

26. Li H, Fan H, Li Y, Shi GY, Ding ZY, Gu ZH, Zhang L. Construction and application of multi-host integrative vector system for xylose-fermenting yeast. FEMS Yeast Res. 2017;17(6)

27. Guo YG, Wu J, Jia H, Chen W, Shao CS, Zhao L, Ma JJ, Li R, Zhong YJ, Fang $\mathrm{F}$, et al. Balancing the expression and production of a heterodimeric protein: recombinant agkisacutacin as a novel antithrombotic drug candidate. Sci Rep-Uk. 2015;5(11730):1-10.

28. Juturu V, Wu JC. Heterologous protein expression in Pichia pastoris: latest research progress and applications. Chembiochem. 2018:19(1):7-21.

29. Waterham HR, Digan ME, Koutz PJ, Lair SV, Cregg JM. Isolation of the Pichia pastoris glyceraldehyde-3-phosphate dehydrogenase gene and regulation and use of its promoter. Gene. 1997;186(1):37-44.

30. Dragosits M, Stadlmann J, Albiol J, Baumann K, Maurer M, Gasser B, Sauer M, Altmann F, Ferrer P, Mattanovich $D$. The effect of temperature on the proteome of recombinant Pichia pastoris. J Proteome Res. 2009:8(3):1380-92.

31. $\mathrm{Yu} \mathrm{H}$, Huang $\mathrm{H}$. Engineering proteins for thermostability through rigidifying flexible sites. Biotechnol Adv. 2014;32(2):308-15.

32. Johnson MR, Wang K, Smith JB, Heslin MJ, Diasio RB. Quantitation of dihydropyrimidine dehydrogenase expression by real-time reverse transcription polymerase chain reaction. Anal Biochem. 2000;278(2):175-84.

\section{Publisher's Note}

Springer Nature remains neutral with regard to jurisdictional claims in published maps and institutional affiliations.

Ready to submit your research? Choose BMC and benefit from:

- fast, convenient online submission

- thorough peer review by experienced researchers in your field

- rapid publication on acceptance

- support for research data, including large and complex data types

- gold Open Access which fosters wider collaboration and increased citations

- maximum visibility for your research: over $100 \mathrm{M}$ website views per year

At $\mathrm{BMC}$, research is always in progress.

Learn more biomedcentral.com/submissions 